\title{
Dietary treatment of the metabolic syndrome - the optimal diet
}

\author{
Gabriele Riccardi* and A. A. Rivellese \\ Department of Clinical and Experimental Medicine, Federico II University Medical School, Naples, Italy
}

\begin{abstract}
The treatment of the metabolic syndrome aims to improve insulin sensitivity and correct/prevent the associated metabolic and cardiovascular abnormalities. Since many individuals with the metabolic syndrome are overweight, dietary treatment should be primarily focused on weight reduction. This approach can improve insulin sensitivity and exert beneficial effects on all the other abnormalities clustering in the syndrome. Insulin sensitivity can also be influenced by diet composition. In this respect, the specific effects of the quality of dietary fat are of great interest, given the considerable evidence in experimental animals that saturated fat in the diet may lead to insulin resistance. In man, there is indirect evidence that a higher saturated fat intake is associated with impaired insulin action. Human studies have also attempted to evaluate the relationship between total fat intake and insulin sensitivity. They are consistent in showing that fat intake is correlated with both plasma insulin values (positively) and insulin sensitivity (negatively). However, these correlations are largely mediated by body weight. Conversely, intervention studies are consistent in showing that when total fat intake is moderately increased (from 20 to $40 \%$ ), no major effect is observed on insulin sensitivity. We have recently undertaken a large, multicentre intervention study in 162 healthy individuals given either a high-saturated-fat or a high-monounsaturated-fat diet for 3 months. It shows that a high-monounsaturated-fat diet significantly improves insulin sensitivity compared to a high-saturated-fat diet. However, this beneficial effect of monounsaturated fat disappears when total fat intake exceeds $38 \%$ of total energy. Independently of its effects on insulin sensitivity, diet composition can influence the factors clustering in the metabolic syndrome. Dietary carbohydrate increases blood glucose levels, particularly in the postprandial period, and consequently also insulin levels and plasma triglycerides. The detrimental effects of a high-carbohydrate diet on plasma glucose/insulin, triglyceride/HDL or fibrinolysis occur only when carbohydrate foods with a high glycaemic index are consumed, while they are abolished if the diet is based largely on fibre-rich, lowglycaemic-index foods. In conclusion, weight reduction is a powerful measure for the treatment of metabolic syndrome. Moreover, the diet for the treatment of the metabolic syndrome should be limited in the intake of saturated fat, while high fibre/low-glycaemic-index foods should be used without specific limitations. Moderate amounts of monounsaturated fat could be permitted as they do not induce detrimental metabolic effects.
\end{abstract}

Metabolic symdrome: Insulin sensitivity: Monounsaturated fat: Diet

The metabolic syndrome is a clustering of metabolic abnormalities and cardiovascular risk factors that occur in individuals with impaired insulin sensitivity. These individuals present two or more of the following conditions: high plasma insulin, high blood pressure, high plasma triglyceride, low HDL, diabetes or impaired blood glucose regulation. The treatment of the metabolic syndrome has to be targeted (i) to improve insulin sensitivity, and (ii) to correct/prevent the associated metabolic and cardiovascular abnormalities.

Usually any therapeutic manoeuvre that improves insulin sensitivity will also have beneficial effects on all the metabolic and cardiovascular abnormalities that are linked with insulin resistance. In contrast, some dietary factors that have no influence on insulin sensitivity are able to modify (in either direction) one or more features of the metabolic syndrome. Therefore a diet to treat individuals with this condition will have some general characteristics, but will have specific properties to take into account the abnormalities present in that individual patient (i.e. hypertension, diabetes, dyslipidaemia).

Most of the individuals affected by the metabolic syndrome are overweight, therefore dietary treatment should be primarily focused on weight reduction. This measure is able, per se, to improve insulin sensitivity and thus exert beneficial effects on all the other abnormalities clustering in the syndrome. Since abdominal obesity is an even stronger predisposing factor for the metabolic syndrome, specific nutritional influences on body fat distribution are important, although not yet exhaustively defined.

It is not necessary to achieve the ideal body weight to 
Table 1. Effects of diet composition on insulin sensitivity

\begin{tabular}{lllclc}
\hline Author & Fat content & Participants & $\begin{array}{c}\text { Duration } \\
\text { (d) }\end{array}$ & Method & Outcome \\
\hline Chen, 1988 & 55 versus 0\% & Healthy & 4 & FSIGT* $^{*}$ & $\downarrow$ \\
Borkman et al. 1991 & 37 versus 22\% & Healthy & 21 & Clamp & $=$ \\
Parillo et al. 1992 & 40 versus 20\% & Type 2 DM & 14 & Clamp & $\uparrow$ \\
Garg et al. 1992 & 50 versus 25\% & Type 2 DM & 21 & Clamp & $=$ \\
Hughes et al. 1995 & 30 versus 20\% & IGT & 84 & Clamp & $=$ \\
\hline
\end{tabular}

${ }^{*}$ FSIGT = frequent sampling intravenous glucose tolerance test (minimal model).

improve the metabolic profile - in most instances a 5-10\% weight reduction is sufficient to induce a clinically relevant effect (Weinstock et al. 1998). In fact, in many studies the improvement of insulin sensitivity due to weight reduction is between 30 and $60 \%$, which is more than that obtained with insulin-sensitizing drugs. The beneficial effects of weight reduction are usually preserved as long as weight is not regained. In this respect, intervention programmes have proven successful up to 2 years, but these studies are still in progress and longer follow-ups are needed.

\section{Diet composition and insulin sensitivity}

Insulin sensitivity can be influenced not only by total energy intake, but also by diet composition. In this respect, the specific effects of the quality of dietary fat are of great interest, as there is considerable evidence in experimental animals that saturated fat in the diet may lead to insulin resistance. In man there is indirect evidence for the same effect: a higher saturated fat intake is associated with impaired insulin action. However, intervention studies on changes in dietary fat quality and insulin sensitivity in humans have so far been inconclusive, perhaps because of the short duration of the study period and the inadequate sample size (Storlien et al. 1996).

Human studies have also attempted to evaluate the relationship between total fat intake and insulin sensitivity. Many epidemiological studies, both cross-sectional and prospective, are now available showing that fat intake is correlated with both plasma insulin values (positively) and insulin sensitivity (negatively). These correlations are largely mediated by body weight, which might explain why in these studies saturated and unsaturated fats (which have identical energy content) show similar relationships with insulin sensitivity. If the effect of total fat intake on body weight is properly accounted for, the relationship between dietary fat and insulin sensitivity becomes less consistent (Storlien et al. 1996).

A more appropriate study design to evaluate the effect of total fat intake on insulin sensitivity, independently of all possible confounders, is the intervention trial. Unfortunately few such studies are available in the literature. However these studies are consistent in showing that when total fat intake is increased from 20 to $40 \%$, no major effect is observed on insulin sensitivity (Table 1) (Borkman et al. 1991; Garg et al. 1992; Parillo et al. 1992; Hughes et al. 1995). Only more pronounced, non-physiological changes as in the Chen Study where fat intake varied from 0 to $55 \%$ - might be able to modify insulin sensitivity (Chen et al. 1988). In the study performed by our group, a reduction of fat intake (monounsaturated fat) counterbalanced by an increased consumption of starchy foods slightly worsened insulin sensitivity. This was probably a consequence of glucotoxicity; our study participants had diabetes, and the increase in the carbohydrate load deteriorated their glycaemic control thus impairing their insulin sensitivity (Parillo et al. 1992).

In order to clarify the complex issue of the effects of dietary fat on insulin sensitivity, we have recently undertaken a large, multi-centre intervention study. The details of the results are still unpublished, but the main outcome has been presented at an international diabetes meeting. In short, this study, undertaken in 162 healthy individuals given either a high-saturated-fat or a high-monounsaturatedfat diet for 3 months, shows that a high monounsaturated fat diet significantly improves insulin sensitivity compared to a high-saturated-fat diet. However this beneficial effect of monounsaturated fat disappears in individuals whose total fat intake exceeds $38 \%$ of total energy (Vessby et al. 1999).

There are few dietary components other than dietary fat that have been shown to influence insulin sensitivity (Table 2 ), and for most of these the available evidence is inconclusive. In summary, there are some indications that alcohol might be beneficial if consumed in limited amounts, whereas it might be detrimental when the intake exceeds 30 g/d (Facchini et al. 1994; Kiechl et al. 1996). Also, it has been suggested that a very high salt intake might impair insulin sensitivity (Donovan et al. 1993).

The effects of high-fibre and/or low-glycaemic-index (GI) foods on insulin sensitivity are particularly controversial. While there is strong evidence supporting their beneficial effects on blood glucose levels, data on their influence on insulin sensitivity are much less clear. In particular, so far no properly designed intervention study with a sufficient sample size has been undertaken to evaluate this issue. Therefore while it can be reasonably hypothesized that dietary fibre and/or low-GI foods might exert a beneficial effect on insulin sensitivity, this cannot be

Table 2. Influence of diet composition on insulin sensitivity

\begin{tabular}{lc}
\hline Diet & Insulin sensitivity \\
\hline Total fat & $\downarrow$ \\
Saturated fat & (threshold?) \\
Fibre and/or low Gl & $\downarrow$ \\
Alcohol & $\uparrow ?$ \\
$\mathrm{NaCl}$ & $\uparrow$ \\
\hline
\end{tabular}


taken for granted (Riccardi \& Rivellese, 1991; Frost et al. 1996).

As to the effect of vitamins on insulin sensitivity, there is no firm evidence of any major role of antioxidant vitamin $\mathrm{C}$ and E (Sanchez-Lugo et al. 1997).

\section{Effects of diet composition on metabolic abnormalities and cardiovascular risk factors clustering in the metabolic syndrome}

Independently of its effects on insulin sensitivity, diet composition can influence each of the factors clustering in the metabolic syndrome. Among the various dietary components, the one that has been most extensively studied is dietary carbohydrate. Since dietary carbohydrate represents a major precursor of plasma glucose, it is obvious that increasing the amount of carbohydrate in the diet will elevate blood glucose levels, particularly in the postprandial period. Plasma glucose concentration represents an important triggering factor for insulin release, therefore a highcarbohydrate diet will also lead to increased insulin levels. The effects of a high-carbohydrate diet will be more pronounced on glucose or insulin levels according to the insulin secretory capacity of the endocrine pancreas (Parillo et al. 1996; Reaven, 1997).

Glucose and lipid metabolism are strongly related, and any derangement of carbohydrate metabolism induced by a high-carbohydrate diet will also increase plasma triglycerides and, possibly, decrease plasma HDL concentrations; this has been clearly shown by a meta-analysis of all studies comparing a high-monounsaturated-fat diet with a high-carbohydrate diet (Fig. 1; Garg 1998). Fibrinolysis is also worsened by a high-carbohydrate diet; this type of diet is associated with an increase of PAI1 in blood (Lopez-Segura et al. 1996).

In short, many features of the metabolic syndrome are worsened by dietary carbohydrate. However, these untoward effects are largely due to an abrupt perturbation, induced by carbohydrate-rich foods, of a metabolic steady state present in the fasting condition. The current hypothesis is that the greater and the more rapid this perturbation, the more pronounced the effects. Therefore all detrimental effects of a high-carbohydrate diet could be minimized if carbohydrate digestion and absorption were slowed down. This has raised much interest in the properties of food that retard food digestion, and has led to a new classification of carbohydrate foods based on their physiological effects in vivo. The GI is the parameter most extensively utilized for this classification. It represents the plasma glucose response to a food, expressed as a percentage of the glucose response to a reference food. Although this index has been criticized for its quantitative approximation, it nevertheless differentiates among the carbohydrate-rich foods the 'fast' and the 'lente' ones, which clinical studies have shown as having diverging effects on most of the metabolic abnormalities and cardiovascular risk factors clustering in the metabolic syndrome (Asp et al. 1993; Jenkins \& Jenkins, 1995).

In particular it is now clear that the detrimental effects of a high-carbohydrate diet on plasma glucose/insulin, triglyceride/HDL or fibrinolysis occur only when carbohydrate foods with a high GI are consumed, while they are abolished if the diet is based largely on fibre-rich, low-GI foods (Table 3) (Riccardi \& Rivellese, 1991; Rivellese et al. 1994; Salméron et al. 1997; Frost et al. 1999).

The advantage of GI is that it allows us to predict the physiological effects of a food better than any other chemical or physical parameter. In this respect, although the fibre content of a food certainly contributes to delaying carbohydrate digestion, it is not the only food factor to have such an effect. Therefore, while foods with a high fibre content are often also characterized by a low GI, there still are some discrepancies between fibre content and GI, as in the case of pasta or parboiled rice which are not particularly fibre rich but have a low GI.

In addition to carbohydrate, other food constituents known to influence the metabolic abnormalities and cardiovascular risk factors clustering in the metabolic syndrome are listed in Table 3. In particular, an excessive alcohol intake

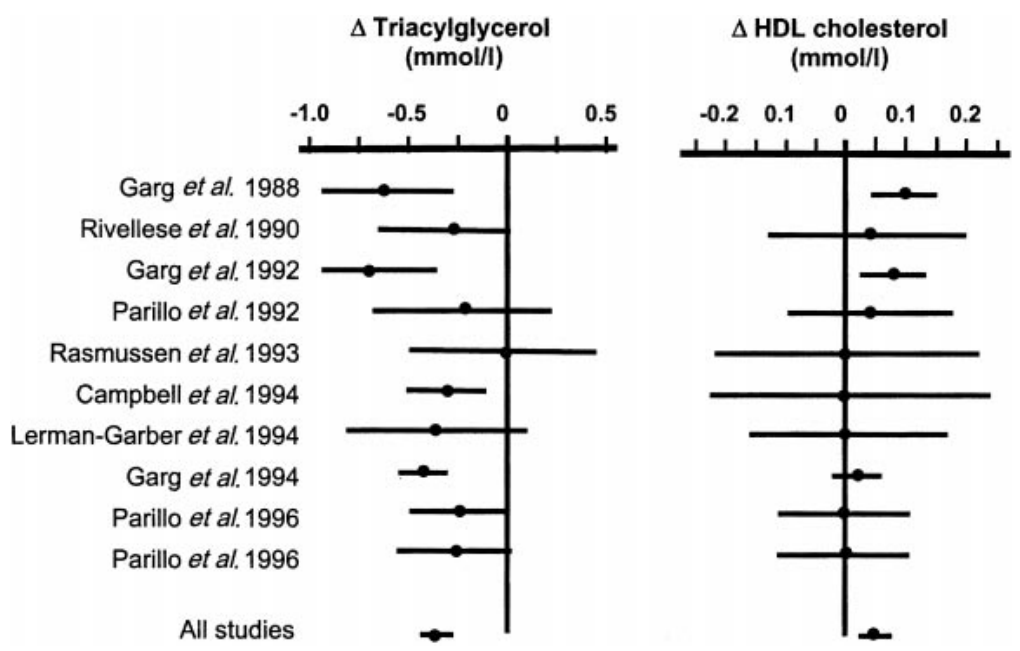

Fig. 1. Mean difference $(95 \% \mathrm{Cl})$ in plasma triglyceride and HDL cholestero concentrations between a high-monounsaturated-fat diet and a high-carbohydrate diet (modified from Garg, 1998). 
Table 3. Effects of dietary components on the cardiovascular risk factors clustering with the metabolic syndrome

\begin{tabular}{lll}
\hline \multirow{2}{*}{ Risk factors } & \multicolumn{2}{c}{ Dietary components with } \\
\cline { 2 - 3 } & \multicolumn{1}{c}{ Beneficial effects } & Detrimental effects \\
\hline Plasma glucose/insulin & Dietary fibre and/or low Gl & High Gl \\
Plasma triglyceride/HDL & Dietary fibre and/or low Gl & High Gl \\
& $\omega-3$ fatty acids & Alcohol \\
Blood pressure & Potassium salts & Sodium chloride \\
& $\omega-3$ fatty acids (?) & Alcohol \\
& & Saturated fat \\
\hline
\end{tabular}

(more than $30 \mathrm{~g} / \mathrm{d}$ ) can increase both plasma triglyceride and blood pressure levels (Kiechl et al. 1996); in addition a high intake of sodium chloride can also elevate blood pressure. In contrast, the $\omega-3$ fatty acids present in fatty fish and some leafy vegetables can reduce plasma triglycerides, whereas their beneficial effects on blood pressure are more controversial. Finally, the potassium salts present in vegetables, legumes and fruit can also contribute to reducing blood pressure, while saturated fat can increase it (Hornstra et al. 1998).

Individuals with the metabolic syndrome are excessively prone to cardiovascular diseases; therefore in defining the 'optimal diet' for these people, the need to reduce plasma cholesterol levels as much as possible and, in particular, low density lipoproteins (LDL), cannot be neglected. In this respect the reduction of saturated fat, which is beneficial for improving insulin sensitivity, is further reinforced as it contributes to lowering LDL. In addition, cholesterol intake has also to be reduced as it influences LDL concentrations and, more generally, cardiovascular risk (Hornstra et al. 1998).

\section{Optimal composition of diet for the metabolic syndrome}

On the basis of what is outlined above, it is not difficult to design a diet for the metabolic syndrome. It should have two basic requirements:

(1) reduced saturated fat;

(2) increased vegetables, legumes, fruit and low-GI starchy foods.

Two additional important features of this diet are a low salt content and a limited alchool intake.

The diet should also take into account both food habits and treatment goals; this would allow more flexibility and thus improve dietary compliance and efficacy (ADA Position Statement, 1996). However, in deciding the proportion of other nutrients it might be appropriate to follow some general guidelines based on available evidence and clinical experience. Protein intake should be similar to that of the rest of the population (allowing some fluctuations) with particular emphasis on vegetable proteins and fish (DNSGEASD, 1995; Linn et al. 1996). About $45 \%$ of the energy intake will be distributed between unsaturated fat and highGI carbohydrate. Since an excessive intake of either one of these nutrients has detrimental metabolic effects, it might be better to indicate upper limits for consumption, although allowing some flexibility. These limits could be set at $25 \%$ energy for high-GI starchy foods and $20 \%$ for monounsaturated fat (plus polyunsaturated fat, which is generally recommended not to exceed $10 \%$ energy). As a consequence, total fat intake could range between 30 and $40 \%$ and total carbohydrate intake between 45 and $55 \%$ (Fig. 2).

Variations within the allowed ranges for carbohydrate and fat may be permitted not only to match (as far as possible) the composition of the habitual diet, but also to consider the specific treatment goals for such individuals. This means increasing either carbohydrate or fat intake in relation to whether the principal goal is weight reduction (in the case of overweight) or limiting the glycaemic load of the diet (in the presence of hyperglycaemia or hypertriglyceridaemia). In the presence of overweight, reducing the fat content might not be sufficient and the total energy intake should also be reduced by decreasing the consumption of all energy-dense foods (including soft drinks and sweets).

\section{Conclusions}

In conclusion, while it is firmly established that weight reduction is a powerful measure for the treatment of the metabolic syndrome, long-term and sufficiently powerful

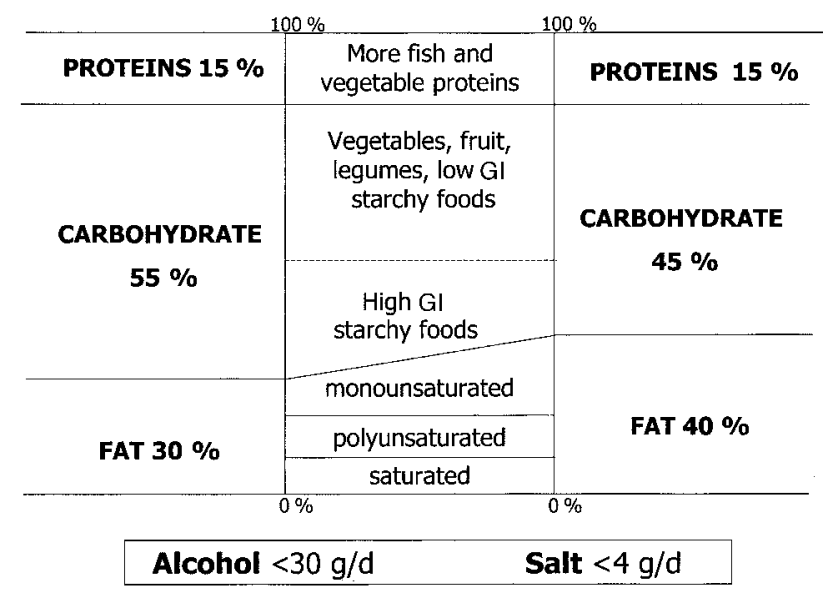

Fig. 2. 'Optimal diet' for the metabolic syndrome. Provided that saturated fat is reduced and most of the carbohydrate-rich foods have a high fibre content or a low GI, some flexibility might be allowed in the amount of fat and carbohydrate, e.g. exchanging about $10 \%$ energy between monounsaturated fat and carbohydrate-rich foods with high Gl. 
(sufficient sample size) intervention studies are still needed to establish how changes in the diet composition can influence insulin sensitivity in humans. At present, on the basis of the best available evidence, the diet for treatment of the metabolic syndrome should be limited in the intake of saturated fat, for its known unfavourable effects on insulin sensitivity and blood pressure, as well as on plasma lipids. Carbohydrate-rich foods with a high GI should also be restricted for their unfavourable effects on the metabolic abnormalities and cardiovascular risk factors clustering in the metabolic syndrome. High fibre/low-GI foods should, instead, be preferentially used without specific limitations. Moderate amounts of monounsaturated fat could be permitted since they do not induce detrimental metabolic effects. If the diet has these characteristics (in addition to a low-salt and moderate alcohol consumption), it may not be necessary to drastically reduce the total amount of fat, as advocated in the past in order to provide cardiovascular disease protection. It is now clear that, with certain limits, it is the quality rather than the total amount of fat that really matters. Moreover, too much emphasis on the need to reduce total fat intake could lead people to increase their carbohydrate consumption. The fact that high-GI foods are often more palatable than low-GI/high-fibre foods could result in an inappropriately high glycaemic load that, in turn, will induce clinically significant untoward effects in people with established metabolic abnormalities. Setting an upper limit for fat consumption around $40 \%$ is both realistic and biologically sound; so far, no clear clinical disadvantage has been demonstrated for diets deriving up to $40 \%$ of energy as fat, provided that the saturated type is kept low. Moreover, since between one-third and one-half of the populations of most western countries derive more than $40 \%$ of energy from fat, the goal of not exceeding this limit is more feasible than any drastic fat reduction which could discourage both patients and physicians from trying to achieve it (Hepp, 1995; Macdiarmid et al. 1996; Heini \& Weinsier, 1997).

Properly designed intervention studies with an adequate sample size should be undertaken in order to evaluate the clinical benefits of an appropriate nutritional approach to treating the metabolic syndrome, possibly as compared with the multi-pharmacological approach presently adopted by most physicians. Unfortunately, for various reasons nutritional studies are not easily funded by either the food industry or institutional bodies. In the absence of clear scientific evidence, individuals with metabolic syndrome will continue to be treated with multiple drug prescriptions that mitigate the symptoms, but are inadequate to treat the disease.

\section{References}

ADA (1996) Nutrition Recommendations and principles for people with diabetes mellitus. Position Statement. Diabetes Care 19 (Suppl. 1), S16-S19.

Asp NG, Bjork I \& Nyman M (1993) Physiological effects of cereal dietary fibre. Carbohydrate Polymers 21, 183-187.

Borkman M, Campbell LV, Chisholm DJ \& Storlien LH (1991) Comparison of the effects on insulin sensitivity of high carbohydrate and high fat diets in normal subjects. Journal of Clinical Endocrinology and Metabolism 72, 432-437.

Campbell LV, Marmot PE, Dyer JA, Borkman M \& Storlien LH (1994) The high-monounsaturated fat diet as a practical alternative for NIDDM. Diabetes Care 17, 177-188.

Chen M, Bergman R \& Porte D (1988) Insulin resistance and betacell dysfunction in aging: the importance of dietary carbohydrate. Journal of Clinical Endocrinology and Metabolism 67, 951-957.

DNSG-EASD (1995) Diabetes and Nutrition Study Group (DNSG) of the European Association for the Study of Diabetes. Recommendations for the nutritional management of patients with diabetes mellitus. Diabetes, Nutrition and Metabolism $\mathbf{8}$, $1-4$.

Donovan DS, Soloman CG, Seely EW et al. (1993) Effect of sodium intake on insulin sensitivity. American Journal of Physiology 264, E730-E734.

Facchini F, Chen Y-DI \& Reaven GM (1994) Light-to-moderate alcohol intake is associated with enhanced insulin sensitivity. Diabetes Care 17, 115-119.

Frost G, Keogh B, Smith D, Akinsanya K \& Leeds A (1996) The effect of low glycemic carbohydrate on insulin and glucose response in vivo and in vitro in patients with coronary heart disease. Metabolism 45, 669-672.

Frost G, Leeds AA, Dore CJ, Madeiros S, Brading S \& Dornhorst A (1999) Glycemic index as a determinant of serum HDLcholesterol concentration. Lancet 353, 1045-1048.

Garg A (1998) High-monounsaturated fat diets for patients with diabetes mellitus: a meta-analysis. American Journal of Clinical Nutrition 67 (Suppl), 577S-582S.

Garg A, Bonanome A, Grundy SM, Zheng ZJ \& Unger RH (1988) Comparison of a high-carbohydrate diet with a highmonounsaturated-fat diet in patients with non-insulin-dependent diabetes mellitus. New England Journal of Medicine 319, 829834.

Garg A, Grundy SM \& Unger RH (1992) Comparison of effects of high and low carbohydrate diets on plasma lipoproteins and insulin sensitivity in patients with mild NIDDM. Diabetes 41, $1278-1285$.

Garg A, Bantle JP, Henry RR et al. (1994) Effects of varying carbohydrate content of diet in patients with non-insulin dependent diabetes mellitus. Journal of the American Medical Association 271, 1421-1428.

Heini AF \& Weinsier RL (1997) Divergent trends in obesity and fat intake patterns: the American paradox. American Journal of Medicine 102, 259-264.

Hepp KD (1995) Food consumption habits in Germany - the clinician's point of view. Metabolism 44 (Suppl. 2), 14-17.

Hornstra G, Barth CA, Galli C, Mensink RP, Mutanen M, Riemersma RA, Roberfroid M, Salminen K, Vansant G \& Vershuren PM (1998) Functional food science and the cardiovascular system. British Journal of Nutrition 80, S113-S146.

Hughes VA, Fiatarone MA, Fielding RA, Ferrara CM, Elahi D \& Evans WJ (1995) Long-term effects of a high-carbohydrate diet and exercise on insulin action in older subjects with impaired glucose tolerance. American Journal of Clinical Nutrition 62 , 426-433.

Jenkins DJA \& Jenkins AL (1995) Nutrition principles and diabetes. A role for 'lente carbohydrate'? Diabetes Care 18, 1491-1498.

Kiechl S, Willeit J, Poewe W, Egger G, Oberhollenzer F, Muggeo M \& Bonora E (1996) Insulin sensitivity and regular alcohol consumption: large, prospective, cross sectional population study (Bruneck Study). British Medical Journal 313, 1040-1044.

Lerman-Garber I, Ichazo-Cerro S, Zamora-Gonzales J, CardosoSaldana G \& Posadas-Romero C (1994) Effect of a high 
monounsaturated-fat diet enriched with avocado in NIDDM patients. Diabetes Care 17, 311-315.

Linn T, Geyer R, Prassek S \& Lauke H (1996) Effect of dietary protein intake on insulin secretion and glucose metabolism in insulin-dependent diabetes mellitus. Journal of Clinical Endocrinology and Metabolism 81, 3938-3943.

Lopez-Segura F, Velasco F, Lopez-Miranda J, Castro P, LopezPedrera R, Blanco A, Jimenez-Pereperez J, Torre A, Trujillo J, Ordovas JM \& Perez-Jimenez F (1996) Monounsaturated fatty acid-enriched diet decreases plasma plasminogen activator inhibitor type 1. Arteriosclerosis, Thrombosis and Vascular Biology 16, 82-88.

Macdiarmid JI, Cade JE \& Blundell JE (1996) High and low fat consumers, their macronutrient intake and body mass index: further analysis of the National Diet and Nutrition Survey of British Adults. European Journal of Clinical Nutrition 50, 505512.

Parillo M, Rivellese A, Ciardullo AV, Capaldo B, Giacco A, Genovese S \& Riccardi G (1992) A high monounsaturated fat/ low carbohydrate diet improves periferal insulin sensitivity in non insulin dependent diabetic patients. Metabolism 41, 13731378.

Parillo M, Giacco R, Ciardullo AV, Rivellese AA \& Riccardi G (1996) Does a high-carbohydrate diet have different effects in NIDDM patients treated with diet alone on hypoglycemic drugs? Diabetes Care 19, 498-500.

Rasmussen OW, Thomsen C, Hansen KW, Vesterlund M, Winther E \& Hermansen K (1993) Effects of blood pressure, glucose, and lipid levels of a high-monounsaturated fat diet compared with a high-carbohydrate diet in NIDDM subjects. Diabetes Care 16, $1565-1571$.

Reaven G (1997) Do high carbohydrate diets prevent the development or attenuate the manifestations (or both) of syndrome
$\mathrm{X}$ ? A viewpoint strongly against. Current Opinion in Lipidology 8, 23-27.

Riccardi G \& Rivellese AA (1991) Effects of dietary fiber and carbohydrate on glucose and lipoprotein metabolism in diabetic patients. Diabetes Care 14, 1115-1125.

Rivellese AA, Giacco R, Genovese S et al. (1990) Effects of changing amount of carbohydrate in diet on plasma lipoproteins and apolipoproteins in type II diabetic patients. Diabetes Care 13, 446-448.

Rivellese AA, Auletta P, Marotta G, Saldalamacchia G, Giacco A, Mastrilli V, Vaccaro O \& Riccardi G (1994) Long term metabolic effects of two dietary methods of treating hyperlipidemia. British Medical Journal 308, 227-231.

Salméron J, Manson JE, Stampfer MJ, Colditz GA, Wing AL \& Willett WC (1997) Dietary fiber, glycemic load, and risk of noninsulin-dependent diabetes mellitus in women. Journal of the American Medical Association 277, 472-477.

Sanchez-Lugo L, Mayer-Davis EJ, Howard G, Selby JV, Ayad MF, Rewers M \& Haffner S, for the IRAS Investigators (1997) Insulin sensitivity and intake of vitamins $E$ and $C$ in African American, Hispanic, and non-Hispanic white men and women: the Insulin Resistance and Atherosclerosis Study (IRAS). Americal Journal of Clinical Nutrition 66, 12241231.

Storlien LH, Baur LA, Kriketas AD, Pan DA, Cosney GJ, Jankins AB, Calvert GD \& Campbell LV (1996) Dietary fats and insulin action. Diabetologia 39, 621-631.

Vessby B, for the Kanwu Study Group (1999) Effect of dietary fat on insulin sensitivity and insulin secretion. The Kanwu Study. Diabetologia 42 (Suppl. 1), A46.

Weinstock RS, Dai H \& Wadden T (1998) Diet and exercise in the treatment of obesity. Effects of three interventions on insulin resistance. Archives of Internal Medicine 158, 2477-2483. 